\title{
Diseño a flexión de vigas de hormigón simplemente apoyadas utilizando lógica difusa
}

\author{
Santamaría J. L.***; Morales L. \\ *Universidad Central del Ecuador, Facultad de Ingeniería, Ciencias \\ Físicas y Matemática, Carrera de Ingeniería Civil, Quito, Ecuador \\ **Autor correspondiente \\ e-mail: jsantamaria@uce.edu.ec
}

Información del artículo

Recibido: julio 2018

aceptado:septiembre 2018

\begin{abstract}
Resumen
Las vigas de hormigón armado son elementos estructurales esenciales, muy utilizadas en toda infraestructura por lo que su diseño se hace recurrente. Diferentes códigos de construcción son empleados para su diseño, mismos que utilizan ecuaciones o ábacos para calcular el acero de refuerzo. El presente estudio muestra una metodología alternativa para determinar el acero de refuerzo de vigas de hormigón armado, simplemente apoyadas, sometidas a cargas uniformemente distribuidas, a través del uso de la teoría de lógica difusa. Un Sistema de Inferencia Lógico (SIL) tipo Sugeno fue desarrollado a partir de datos provenientes del diseño realizado con el código de construcción ACI 318 -14 de los Estados Unidos y el uso del método de agrupamiento sustractivo de datos y ANFIS. Los resultados indican que el modelo difuso tipo Sugeno es capaz de predecir nuevos datos de una manera adecuada $\left(\mathrm{R}^{2}=95.5 \%\right)$ y que podría ser utilizado para el diseño de vigas por cuanto el área del acero de refuerzo colocado efectivamente en una viga no necesita ser exactamente igual al área de acero calculada. Además, el método propuesto es muy flexible y puede ser extendido al diseño de otros elementos estructurales siempre y cuando se disponga de datos reales o experimentales para realizar la modelación difusa.
\end{abstract}

Palabras clave: diseño de vigas de hormigón, agrupamiento sustractivo de datos, ANFIS, lógica difusa

\begin{abstract}
Concrete reinforced beams are essential structural elements that are commonly used in every infrastructure, which makes their design to become recurrent. Several construction building codes are utilized for designing beams using equations or correlations to compute the area of steel needed. The present study illustrates an alternative framework in order to determine the area of steel for reinforced simple supported concrete beams, subjected to uniform loads through the use of fuzzy set theory. A Sugeno type Fuzzy Inference System (SIL) was developed based on actual data resulting from using the ACI 318-14, a United States building code, and the use of subtractive clustering method and ANFIS. The results indicate that the Sugeno type fuzzy model is able to predict new data very well $\left(\mathrm{R}^{2}=95.5 \%\right)$, and that it could actually be used for designing concrete beams since the actual area of steel placed in the beam is not necessarily exactly the same as the calculated area. Furthermore, the proposed methodology could be extended to the design of other structural elements as long as real or experimental data are available for performing the fuzzy modeling.
\end{abstract}

Keywords: Concrete beam design, concrete, subtractive clustering method, ANFIS, fuzzy logic. 


\section{Introducción}

las vigas de hormigón armado son elementos estructurales muy utilizados en la construcción de proyectos de ingeniería ya que comprenden gran parte de toda infraestructura física, especialmente aquella destinada a brindar servicios de vivienda. Razón por la cual se requiere que su diseño se lo lleve a cabo de una manera muy frecuente. Una viga se la puede definir como un elemento estructural que soporta principalmente cargas o fuerzas que actúan perpendicular a su eje longitudinal, soportando esfuerzos de flexión generados por momentos actuantes en sus extremos. Las vigas al ser parte de una estructura son de vital importancia en el funcionamiento deseado de la misma y es por esta razón que fueron escogidas en el presente estudio.

Diferentes tipos de vigas pueden ser utilizadas en la construcción tomando en cuenta su sección transversal (rectangulares, sección “T” o "I"), tipo de apoyo (en cantiléver, simplemente apoyadas o empotradas) y el tipo de carga actuante sobre ellas (carga puntual o uniformemente distribuida). Para efectos de ilustrar el procedimiento de diseño de vigas de hormigón utilizando lógica difusa, se escogieron vigas rectangulares simplemente apoyadas que soportan cargas distribuidas triangulares, rectangulares y trapezoidales. Sin embargo, la metodología empleada en el presente estudio es aplicable para cualquier tipo de elemento estructural que se considere.

Para realizar el diseño estructural de un elemento de hormigón armado se utilizan procedimientos o normativas, llamados códigos de construcción, que han sido previamente establecidos y aceptados y son de aplicación y uso obligatorio dentro de una determinada zona geográfica. Por ejemplo, en el Ecuador se utiliza la Norma Ecuatoriana de Construcción (NEC), mismo que está basado en el código de los Estados Unidos (ACI 318 -14). Cuando se calcula el área de acero de refuerzo que se debe colocar en una sección transversal de un elemento estructural, en este caso una viga rectangular, se obtienen resultados "exactos", con varias cifras decimales. Todo esto en razón de que dichos resultados provienen de la evaluación de ecuaciones formuladas para el efecto.
Sin embargo, en la práctica, dichos resultados obtenidos del diseño no se los puede colocar en su cantidad exacta ya que dichas áreas de acero de refuerzo calculadas deben ser convertidas a su equivalente de área de varillas comerciales (disponibles en el mercado). Dicha área efectiva de acero que será colocada en la viga es un valor aproximado del área calculada. De hecho, el área de acero seleccionada es un valor cercano al calculado y, preferiblemente, estará por encima de dicho valor (mayor al calculado). Lo que significa que la cuantía de acero colocada no es precisa sino aproximada.

La teoría de lógica difusa es una herramienta idónea cuando se habla de valores aproximados y no precisos. Si se considera el uso de lógica difusa en problemas de ingeniería se debe tomar en consideración la tolerancia a la imprecisión [1]. Existen muchos casos en los que ser preciso no influye determinantemente en el resultado. De hecho, estar cercano al valor calculado es usualmente suficiente para conseguir el objetivo propuesto. Además, se hace necesario tomar en consideración que los códigos de construcción incluyen los denominados factores de seguridad en sus cálculos y resultados; lo cual proporciona un rango de seguridad en los diseños obtenidos. En consecuencia, el uso de la teoría de lógica difusa no influiría significativamente en los resultados finales por cuanto no se usan los valores exactos de diseño, sino aproximados.

El presente estudio tiene por objeto presentar una metodología alternativa para estimar la cuantía de acero de refuerzo () en vigas de hormigón rectangulares simplemente armadas, simplemente apoyadas, y sometidas a cargas distribuidas de tipo triangular, rectangular y trapezoidal a través de la utilización de la teoría de lógica difusa. Un modelo de inferencia lógico capaz de predecir cantidades de acero de refuerzo para vigas fue desarrollado utilizando el método automático para sistemas de inferencia lógicos conocido como: Adaptive Neuro Fuzzy Inference Systems (ANFIS) según sus siglas iniciales del idioma inglés. Dicho procedimiento emplea datos reales sean estos calculados o experimentales del tipo entrada - salida (I/O), conocidos como conocimiento 
previo para crear funciones de membresía (FMs) y reglas difusas del tipo condicional (If - then) que permiten estimar o predecir nuevos datos.

\subsection{Teoría de lógica difusa}

La teoría de lógica difusa es una teoría relativamente nueva que fue desarrollada por Zadeh en [2] con el fin de explicar cómo la imprecisión o incertidumbre puede ser manejada en la solución de problemas. De hecho, lógica difusa es una poderosa herramienta que puede ser utilizada para entender sistemas complejos donde no se dispone de funciones matemáticas [1]. Lógica difusa es una herramienta muy efectiva que permite relacionar datos de entrada con datos de salida y brindar información que permite ganar conocimiento del sistema que se está estudiando. Esta teoría emplea Sistemas de Inferencia Lógicos (SILs) denominados Fuzzy Inference Systems (FIS) por sus siglas en idioma inglés, los cuales incorporan variables lingüísticas relacionadas a través del uso de las denominadas reglas difusas del tipo antecedente - consecuente (If - then) comúnmente utilizadas por las personas.

\subsection{Sistema de inferencia lógico (SIL)}

Los SILs o también llamados modelos difusos, son conocidos como funciones universales no lineales aproximadas capaces de predecir o estimar nuevos datos basados en conocimiento previo [1]. Un SIL está formado por dos partes fundamentales: 1) Conocimiento base, y 2) Mecanismo de inferencia (ver Fig. 1) y son capaces de estimar un resultado mediante el uso de algoritmos de razonamiento aproximado. El conocimiento base comprende el conocimiento previo que incluye datos de entrada y salida (I/O); así como también, la información disponible resultante de la observación del sistema y las reglas difusas que relacionan las variables consideradas. Con respecto al mecanismo de inferencia lógico, los métodos más utilizados son los de Mamdani [3,4], Sugeno [5] y Tsukamoto [6]. De los mencionados procedimientos, solamente los métodos de Sugeno y Tsukamoto producen como resultados valores no difusos (valores discretos).

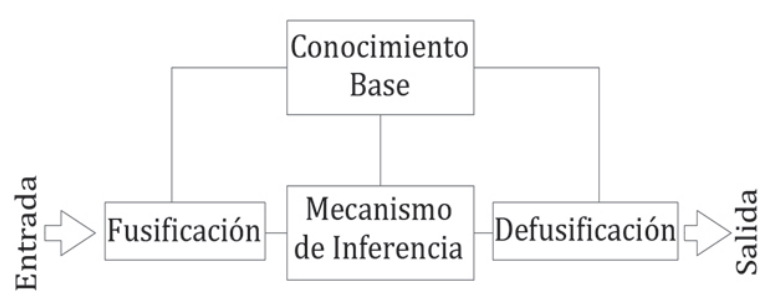

Figura 1. Funcionamiento del Sistema de Inferencia Lógico (SIL).

El razonamiento lógico que es realizado por los SILs se lo lleva a cabo de la siguiente manera según [7]. Primeramente, se asigna los llamados grados de membresía a los valores o datos de entrada mediante la utilización de las funciones de membresía (FMs) y a este proceso se lo conoce como fusificación. Luego se combinan los grados de membresía a través de una operación difusa (T-norm) con el fin de obtener los pesos respectivos llamados "firing strengths" en idioma inglés. Después, se genera los valores consecuentes correspondientes a cada regla difusa basados en los pesos calculados, para finalmente compilar todos los consecuentes y producir un valor discreto.

\subsection{Funciones de membresía (FMs) y reglas difusas (If-then)}

Una FM se la puede definir como una función matemática que describe a un conjunto difuso y se la utiliza para asignar grados de membresía a un determinado elemento. Las formas de las FMs pueden ser variadas: triangular, trapezoidal o gaussiana entre otras; sin embargo, la característica de dichas curvas es que su altura sea la unidad como se indica en la Fig. 2. Las reglas difusas son del tipo condicional de la forma If - then, donde e son los nombres de los conjuntos difusos, mismos que son caracterizados por sus respectivas FMs. Si utilizamos el mecanismo de inferencia lógico Sugeno [5], se tiene conjuntos difusos solo en la premisa () de la regla difusa del tipo If - then, mientras que la parte consecuente () está descrita por una función matemática no difusa. Por ejemplo, la expresión "si el esfuerzo de compresión del hormigón es alto (), entonces la relación agua-cemento es W/C ()". En este caso se utiliza un conjunto difuso que corresponde al esfuerzo de compresión (variable), donde se puede observar cómo se in- 
cluye el uso de términos lingüísticos comúnmente usados por las personas como "alto", el cual es caracterizado por una función, que es una FM. En lo que respecta al valor consecuente $(\mathrm{W} / \mathrm{C})$, este es un valor discreto.

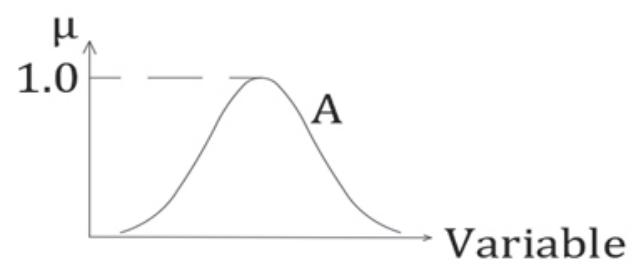

Figura 2. Función de membresía (FM) tipo gaussiana.

\section{Metodología}

La Fig. 3 ilustra la metodología que será aplicada a fin de estimar el acero de refuerzo en vigas simplemente apoyadas, de sección rectangular, que trabajan a flexión y que están sometidas a cargas uniformemente distribuidas.

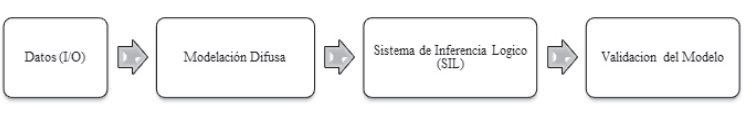

Figura 3. Metodología de la investigación.

\subsection{Datos $(I / O)$}

Para el cálculo del acero de refuerzo () se utilizaron vigas de sección rectangular, comúnmente utilizadas y diseñadas en el medio, cuyas características son: base () entre $25 \mathrm{~cm}$ y $40 \mathrm{~cm}$; altura () entre $40 \mathrm{~cm}$ y $70 \mathrm{~cm}$; carga distribuida () entre 3.5 $\mathrm{T} / \mathrm{m}$ a $13.5 \mathrm{~T} / \mathrm{m}$ de tipo rectangular, trapezoidal y triangular, y luces () entre $3 \mathrm{~m}$ y $10 \mathrm{~m}$, según se observa en la Fig. 4. El valor de corresponde a los extremos de la carga trapezoidal medida desde el apoyo. El código empleado para el diseño de las vigas de hormigón fue el Código de Construcción ACI 318-14 de los Estados Unidos y los parámetros de diseño se encuentran compilados en la Tabla 1 . Un total de 105 vigas fueron diseñadas para generar datos del tipo de entrada - salida (I/O), de los cuales se escogió los datos para entrenar (TR) y chequear $(\mathrm{CH})$ el modelo ANFIS (ver Tabla 2). 75 y 30 filas de datos fueron escogidas al azar para entrenar y chequear el referido modelo difuso que será utilizado para generar un sistema de inferencia lógico (SIL) tipo Sugeno capaz de estimar el necesario para resistir las condiciones prestablecidas de diseño.

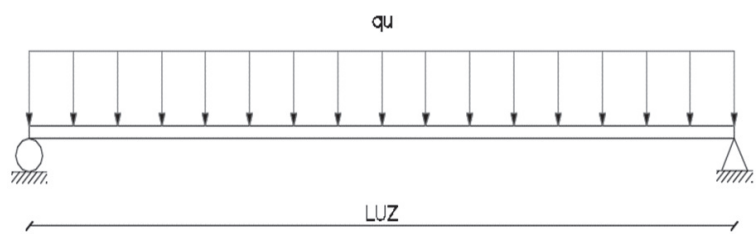

Viga Tipo 1

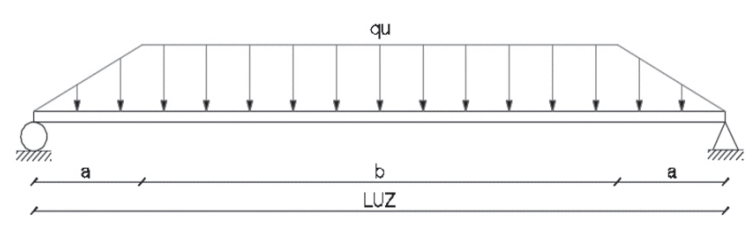

Viga Tipo 2

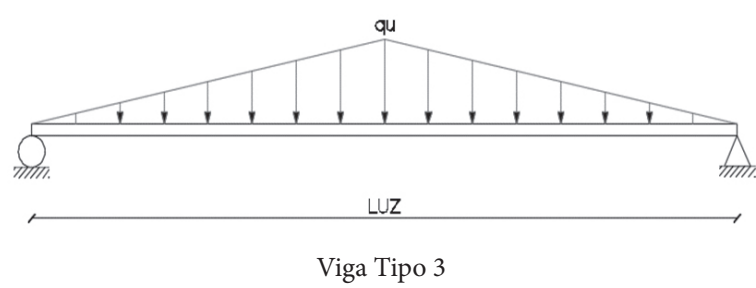

Figura 4. Esquema de vigas sometidas a cargas distribuidas.

Tabla 1. Parámetros de diseño

\begin{tabular}{ll}
\hline Parámetro & Valor \\
\hline $\begin{array}{l}\text { Resistencia a la compresión del } \\
\text { hormigón }\left(f^{\prime} c\right)=\end{array}$ & $21 \mathrm{MPa}$ a $35 \mathrm{MPa}$ \\
$\begin{array}{l}\text { Límite de fluencia del acero de } \\
\text { refuerzo }(f y)=\end{array}$ & $4200 \mathrm{Kg} / \mathrm{cm}^{2}$ \\
$\begin{array}{l}\text { Deformación mínima del acero } \\
\text { a mas traccionado }(\xi t)=\end{array}$ & $0.005 \mathrm{~mm} / \mathrm{mm}$ \\
$\begin{array}{l}\text { Deformación última del } \\
\text { hormigón }(\xi c u)=\end{array}$ & $0.003 \mathrm{~mm} / \mathrm{mm}$ \\
$\begin{array}{l}\text { Deformación unitaria del acero } \\
(\xi s)=\end{array}$ & $0.0021 \mathrm{~mm} / \mathrm{mm}$ \\
$\begin{array}{l}\text { Recubrimiento }= \\
\text { Estribo }=\end{array}$ & $4 \mathrm{~cm}$ \\
\hline
\end{tabular}


Tabla 2. Datos utilizados para la modelación difusa

\begin{tabular}{|c|c|c|c|c|c|c|c|c|c|}
\hline \multirow{2}{*}{$\mathbf{N}^{\circ}$} & \multirow{2}{*}{$\begin{array}{l}\text { Viga } \\
\text { Tipo }\end{array}$} & f'c & $b$ & $h$ & luz & $a$ & $q u$ & As & \multirow{2}{*}{ Dato } \\
\hline & & $\begin{array}{l}(\mathrm{Kg} / \\
\left.\mathrm{cm}^{2}\right)\end{array}$ & (m) & $(\mathbf{m})$ & (m) & (m) & $(\mathrm{Kg} / \mathrm{m})$ & $\left(\mathrm{cm}^{2}\right)$ & \\
\hline 1 & 1 & 210 & 25 & 40 & 4 & 0.0 & 4 & 6.88 & TR \\
\hline 2 & 1 & 210 & 25 & 45 & 5 & 0.0 & 4.5 & 11.00 & TR \\
\hline 3 & 1 & 210 & 30 & 45 & 6 & 0.0 & 4 & 14.26 & TR \\
\hline 4 & 1 & 210 & 30 & 55 & 7 & 0.0 & 4.5 & 17.28 & $\mathrm{CH}$ \\
\hline 5 & 1 & 210 & 30 & 60 & 8 & 0.0 & 4 & 18.05 & TR \\
\hline 6 & 1 & 210 & 25 & 40 & 4 & 0.0 & 5 & 8.87 & $\mathrm{CH}$ \\
\hline 7 & 1 & 210 & 30 & 45 & 5 & 0.0 & 5.5 & 13.50 & TR \\
\hline 8 & 1 & 240 & 25 & 40 & 4 & 0.0 & 4 & 6.78 & TR \\
\hline 9 & 1 & 240 & 25 & 40 & 3 & 0.0 & 5 & 4.64 & TR \\
\hline 10 & 1 & 240 & 30 & 45 & 5 & 0.0 & 6 & 14.60 & $\mathrm{CH}$ \\
\hline 11 & 1 & 240 & 30 & 55 & 6 & 0.0 & 7 & 19.75 & TR \\
\hline 12 & 1 & 240 & 35 & 60 & 7 & 0.0 & 8 & 28.42 & $\mathrm{TR}$ \\
\hline 13 & 1 & 240 & 30 & 45 & 4 & 0.0 & 9 & 13.92 & TR \\
\hline 14 & 1 & 240 & 35 & 55 & 5 & 0.0 & 11 & 21.29 & $\mathrm{CH}$ \\
\hline 15 & 1 & 280 & 25 & 40 & 4 & 0.0 & 4 & 6.69 & TR \\
\hline 16 & 1 & 280 & 35 & 55 & 6 & 0.0 & 10 & 28.48 & $\mathrm{CH}$ \\
\hline 17 & 1 & 280 & 35 & 60 & 7 & 0.0 & 8 & 27.56 & TR \\
\hline 18 & 1 & 280 & 30 & 55 & 8 & 0.0 & 4 & 19.59 & TR \\
\hline 19 & 1 & 280 & 35 & 65 & 9 & 0.0 & 6 & 31.49 & TR \\
\hline 20 & 1 & 280 & 40 & 70 & 8 & 0.0 & 10 & 38.29 & $\mathrm{CH}$ \\
\hline 21 & 1 & 280 & 25 & 45 & 7 & 0.0 & 3.5 & 17.24 & TR \\
\hline 22 & 1 & 300 & 25 & 40 & 4 & 0.0 & 4 & 6.66 & TR \\
\hline 23 & 1 & 300 & 30 & 50 & 6 & 0.0 & 6.5 & 20.12 & $\mathrm{TR}$ \\
\hline 24 & 1 & 300 & 25 & 40 & 5 & 0.0 & 5 & 14.09 & $\mathrm{CH}$ \\
\hline 25 & 1 & 300 & 35 & 60 & 8 & 0.0 & 7.5 & 34.64 & $\mathrm{TR}$ \\
\hline 26 & 1 & 300 & 25 & 45 & 4.5 & 0.0 & 8 & 15.87 & $\mathrm{CH}$ \\
\hline 27 & 1 & 300 & 30 & 50 & 7 & 0.0 & 5 & 21.23 & TR \\
\hline 28 & 1 & 300 & 30 & 55 & 5.5 & 0.0 & 9.5 & 22.15 & TR \\
\hline 29 & 1 & 350 & 25 & 40 & 4 & 0.0 & 4 & 6.59 & $\mathrm{CH}$ \\
\hline 30 & 1 & 350 & 30 & 50 & 5 & 0.0 & 10 & 21.20 & TR \\
\hline 31 & 1 & 350 & 30 & 45 & 6 & 0.0 & 6 & 20.98 & TR \\
\hline 32 & 1 & 350 & 30 & 55 & 7 & 0.0 & 8 & 31.12 & $\mathrm{TR}$ \\
\hline 33 & 1 & 350 & 35 & 65 & 8 & 0.0 & 9 & 37.18 & $\mathrm{CH}$ \\
\hline 34 & 1 & 350 & 40 & 65 & 9 & 0.0 & 8.5 & 44.80 & TR \\
\hline 35 & 1 & 350 & 30 & 55 & 5.5 & 0.0 & 12 & 28.38 & TR \\
\hline 36 & 2 & 210 & 25 & 40 & 4.5 & 1.1 & 4.5 & 9.33 & TR \\
\hline 37 & 2 & 210 & 25 & 45 & 5.5 & 1.4 & 4 & 10.82 & $\mathrm{CH}$ \\
\hline 38 & 2 & 210 & 35 & 60 & 6.5 & 1.6 & 8 & 21.99 & TR \\
\hline 39 & 2 & 210 & 35 & 60 & 7.5 & 1.9 & 6.5 & 24.17 & TR \\
\hline 40 & 2 & 210 & 35 & 60 & 8.5 & 2.1 & 4.5 & 21.01 & $\mathrm{CH}$ \\
\hline 41 & 2 & 210 & 25 & 40 & 3.5 & 0.9 & 6 & 7.29 & TR \\
\hline 42 & 2 & 210 & 30 & 50 & 5 & 1.3 & 7 & 13.75 & TR \\
\hline 43 & 2 & 240 & 25 & 40 & 4.5 & 1.1 & 5 & 10.32 & $\mathrm{CH}$ \\
\hline 44 & 2 & 240 & 25 & 40 & 3.5 & 0.9 & 5.5 & 6.52 & TR \\
\hline
\end{tabular}

\begin{tabular}{|c|c|c|c|c|c|c|c|c|c|}
\hline 45 & 2 & 240 & 30 & 50 & 5.5 & 1.4 & 7 & 16.79 & TR \\
\hline 46 & 2 & 240 & 35 & 60 & 6.5 & 1.6 & 8.5 & 23.07 & TR \\
\hline 47 & 2 & 240 & 40 & 65 & 7.5 & 1.9 & 9 & 29.93 & $\mathrm{CH}$ \\
\hline 48 & 2 & 240 & 25 & 45 & 3.5 & 0.9 & 9.5 & 10.13 & TR \\
\hline 49 & 2 & 240 & 30 & 55 & 4.5 & 1.1 & 12 & 17.08 & TR \\
\hline 50 & 2 & 280 & 25 & 45 & 5 & 1.3 & 5.5 & 11.99 & TR \\
\hline 51 & 2 & 280 & 30 & 55 & 5.5 & 1.4 & 11 & 24.07 & $\mathrm{CH}$ \\
\hline 52 & 2 & 280 & 35 & 55 & 6.5 & 1.6 & 8.5 & 25.60 & TR \\
\hline 53 & 2 & 280 & 30 & 55 & 7.5 & 1.9 & 5 & 19.75 & $\mathrm{TR}$ \\
\hline 54 & 2 & 280 & 35 & 65 & 8.5 & 2.1 & 7.5 & 32.51 & $\mathrm{CH}$ \\
\hline 55 & 2 & 280 & 35 & 55 & 6 & 1.5 & 11 & 28.77 & TR \\
\hline 56 & 2 & 280 & 25 & 40 & 3.5 & 0.9 & 4 & 4.59 & TR \\
\hline 57 & 2 & 300 & 25 & 40 & 4 & 1.0 & 6 & 9.42 & $\mathrm{TR}$ \\
\hline 58 & 2 & 300 & 30 & 45 & 5.5 & 1.4 & 7 & 19.01 & $\mathrm{CH}$ \\
\hline 59 & 2 & 300 & 30 & 45 & 6.5 & 1.6 & 5 & 18.96 & $\mathrm{CH}$ \\
\hline 60 & 2 & 300 & 35 & 60 & 7.5 & 1.9 & 8 & 28.91 & TR \\
\hline 61 & 2 & 300 & 30 & 45 & 5 & 1.3 & 9 & 20.44 & TR \\
\hline 62 & 2 & 300 & 30 & 50 & 6.5 & 1.6 & 6.5 & 21.93 & $\mathrm{TR}$ \\
\hline 63 & 2 & 300 & 35 & 55 & 9 & 2.3 & 5 & 29.15 & $\mathrm{TR}$ \\
\hline 64 & 2 & 350 & 35 & 60 & 8 & 2.0 & 8.5 & 35.39 & TR \\
\hline 65 & 2 & 350 & 30 & 55 & 5.5 & 1.4 & 11 & 23.17 & $\mathrm{CH}$ \\
\hline 66 & 2 & 350 & 35 & 55 & 7.5 & 1.9 & 7.5 & 29.75 & TR \\
\hline 67 & 2 & 350 & 30 & 55 & 6.5 & 1.6 & 9 & 27.05 & TR \\
\hline 68 & 2 & 350 & 35 & 65 & 8 & 2.0 & 9.5 & 35.78 & TR \\
\hline 69 & 2 & 350 & 40 & 65 & 8.5 & 2.1 & 9.5 & 40.31 & TR \\
\hline 70 & 2 & 350 & 30 & 55 & 5.5 & 1.4 & 13.5 & 29.44 & $\mathrm{CH}$ \\
\hline 71 & 3 & 210 & 25 & 40 & 5 & 2.5 & 5 & 0.00 & $\mathrm{CH}$ \\
\hline 72 & 3 & 210 & 25 & 45 & 5.5 & 2.8 & 6 & 10.82 & TR \\
\hline 73 & 3 & 210 & 30 & 55 & 7.5 & 3.8 & 6 & 21.99 & TR \\
\hline 74 & 3 & 210 & 35 & 55 & 8 & 4.0 & 6 & 24.17 & $\mathrm{CH}$ \\
\hline 75 & 3 & 210 & 30 & 55 & 8.5 & 4.3 & 5 & 21.01 & TR \\
\hline 76 & 3 & 210 & 25 & 45 & 5.5 & 2.8 & 6 & 7.29 & TR \\
\hline 77 & 3 & 210 & 30 & 50 & 6 & 3.0 & 7 & 13.75 & TR \\
\hline 78 & 3 & 240 & 25 & 40 & 4.5 & 2.3 & 6 & 10.32 & $\mathrm{TR}$ \\
\hline 79 & 3 & 240 & 25 & 40 & 4.5 & 2.3 & 6.5 & 6.52 & $\mathrm{CH}$ \\
\hline 80 & 3 & 240 & 30 & 45 & 6 & 3.0 & 7 & 16.79 & $\mathrm{CH}$ \\
\hline 81 & 3 & 240 & 30 & 50 & 6.5 & 3.3 & 8 & 23.07 & TR \\
\hline 82 & 3 & 240 & 35 & 65 & 8.5 & 4.3 & 9.5 & 29.93 & TR \\
\hline 83 & 3 & 240 & 30 & 50 & 5 & 2.5 & 11 & 10.13 & TR \\
\hline 84 & 3 & 240 & 30 & 55 & 5.5 & 2.8 & 12.5 & 17.08 & TR \\
\hline 85 & 3 & 280 & 25 & 40 & 5.5 & 2.8 & 5 & 11.99 & TR \\
\hline 86 & 3 & 280 & 35 & 55 & 7 & 3.5 & 11 & 24.07 & $\mathrm{CH}$ \\
\hline 87 & 3 & 280 & 35 & 55 & 7.5 & 3.8 & 9.5 & 25.60 & TR \\
\hline 88 & 3 & 280 & 35 & 55 & 9.5 & 4.8 & 6 & 19.75 & $\mathrm{TR}$ \\
\hline 89 & 3 & 280 & 35 & 65 & 10 & 5.0 & 7.5 & 32.51 & TR \\
\hline 90 & 3 & 280 & 35 & 65 & 8.5 & 4.3 & 11 & 28.77 & TR \\
\hline 91 & 3 & 280 & 30 & 50 & 8.5 & 4.3 & 4.5 & 4.59 & $\mathrm{CH}$ \\
\hline 92 & 3 & 300 & 25 & 40 & 5 & 2.5 & 5.5 & 9.42 & $\mathrm{CH}$ \\
\hline
\end{tabular}




\begin{tabular}{cccccccccc}
\hline 93 & 3 & 300 & 30 & 50 & 6.5 & 3.3 & 8.5 & 19.01 & TR \\
\hline 94 & 3 & 300 & 30 & 50 & 6.5 & 3.3 & 6.5 & 18.96 & TR \\
\hline 95 & 3 & 300 & 35 & 60 & 9 & 4.5 & 8.5 & 28.91 & TR \\
\hline 96 & 3 & 300 & 25 & 45 & 5 & 2.5 & 9 & 20.44 & TR \\
\hline 97 & 3 & 300 & 30 & 55 & 8.5 & 4.3 & 6.5 & 21.93 & TR \\
\hline 98 & 3 & 300 & 30 & 55 & 6.5 & 3.3 & 11.5 & 29.15 & CH \\
\hline 99 & 3 & 350 & 25 & 40 & 4.5 & 2.3 & 5.5 & 35.39 & TR \\
\hline & 3 & 350 & 30 & 55 & 6.5 & 3.3 & 11 & 23.17 & TR \\
\hline & 3 & 350 & 30 & 45 & 7 & 3.5 & 7 & 29.75 & TR \\
\hline & 3 & 350 & 30 & 55 & 7.5 & 3.8 & 9.5 & 27.05 & CH \\
\hline & 3 & 350 & 40 & 65 & 9.5 & 4.8 & 11 & 35.78 & TR \\
\hline & 3 & 350 & 40 & 65 & 10 & 5.0 & 10 & 40.31 & CH \\
\hline & 3 & 350 & 30 & 50 & 6 & 3.0 & 13 & 29.44 & TR \\
\hline
\end{tabular}

\subsection{Modelación difusa}

La modelación difusa comprende el procedimiento para desarrollar un SIL. Existen dos enfoques para crear modelos difusos [8]. El primero es llamado enfoque directo y consiste en crear un SIL basado únicamente en el conocimiento del técnico o experto quien conoce muy bien el funcionamiento de un sistema. En este caso el experto elabora las funciones de membresía, las reglas difusas, escoge un SIL apropiado y evalúa el modelo difuso. El segundo enfoque es conocido como identificación del sistema y se basa en la utilización de datos del tipo I/O reales a fin de generar de una manera automatizada un SIL tipo Sugeno. En el presente estudio se utilizó esta técnica a fin de estimar el de una viga simplemente armada y simplemente apoyada sometida a cargas uniformemente distribuidas.

\subsection{Identificación del sistema}

Para la realización de la identificación del sistema se requiere del cumplimiento de dos fases que son la identificación de la estructura y la identificación de parámetros [9]. La primera fase consiste en establecer las particiones o agrupamiento de los datos I/O, el número y las reglas difusas de la forma If - then. Los centros de los grupos de datos (clústeres) son identificados mediante el método de agrupamiento sustractivo [10] y son los que determinan el número de reglas difusas y las funciones de membresía que constituyen los antecedentes. En la segunda fase se realiza el ajuste de los parámetros del modelo a fin de minimizar errores a través de ANFIS.

\subsubsection{Identificación de la estructura}

Como se mencionó anteriormente, agrupamiento sustractivo [10] es el método de agrupamiento utilizado para determinar el número de reglas difusas y las funciones de membresía. En este método, cualquier punto es considerado como un centro del grupo o clúster, reduciendo la carga computacional significativamente, aun para un número grande de variables de entrada. Este método es rápido ya que no envuelve optimización no lineal y es recomendado cuando no se conoce el número posible de centros de los clústeres. Por las razones antes mencionadas se utilizará este procedimiento en la presente investigación.

\subsubsection{Agrupamiento sustractivo}

En el presente método [10], cada punto de datos es considerado como un potencial centro del clúster y el valor potencial () de cada punto () está definido por (1). Un punto de datos con muchos puntos alrededor tendrá alto valor potencial y el punto con mayor valor potencial será asignado el primer centro. El valor de se lo puede calcular con (2), donde , una constante positiva, es el radio de influencia del centro del clúster. Valores grandes de producen menos centros de clústeres mientras que valores pequeños generan más centros. Este parámetro es especificado por el usuario y debe ser basado en los resultados que se produzcan de acuerdo a lo deseado. Luego los valores potenciales de los puntos restantes son actualizados con respecto al primer clúster utilizando (3), donde $x_{1}^{*}$ es el primer clúster identificado con su correspondiente valor potencial, $P_{1}^{*}$. El valor de $\beta$ está definido por (4), donde $r_{b}$ es una constante positiva definida por (5) y representa el radio del vecindario que tiene reducciones medibles. El valor de $\eta$ es conocido como "squash factor" y 1.5 es un valor típico que asegura que los centros de los clústeres no estén muy cerca unos de otros; sin embargo, este valor deberá ser analizado de acuerdo al caso.

$$
P i=\sum_{j=1}^{n} e^{-\alpha\left\|x_{i}-x_{j}\right\|^{2}}
$$




$$
\begin{gathered}
\alpha=\frac{4}{r_{a}{ }^{2}} \\
P_{i} \Leftarrow P_{i}-P_{1}^{*} e^{-\beta\left\|x_{i}-x_{1}^{*}\right\|^{2}} \\
\beta=\frac{4}{r_{b}{ }^{2}} \\
r_{b}=\eta * r_{a}
\end{gathered}
$$

Luego de que se han calculado todos los valores potenciales correspondientes con (3), el punto con el más alto valor potencial llega a ser segundo clúster. Se procede de la misma manera para actualizar los siguientes valores potenciales, pero utilizando el segundo clúster y así sucesivamente hasta obtener todos los clústeres según (6), donde $x_{k}^{*}$ y $P_{k}^{*}$ son el clúster $\mathrm{k}^{\text {th }}$ con su respectivo valor potencial.

$$
P_{i} \Leftarrow P_{i}-P_{k}^{*} e^{-\beta\left\|x_{i}-x_{k}^{*}\right\|^{2}}
$$

Como se mencionó anteriormente, el número de clústeres identificados determina el número de FMs y el número total de reglas difusas a utilizarse. Los parámetros de cada FM gaussiana están dados por el centro de cada clúster; mientras que el valor de la desviación estándar está definido por (7).

$$
\sigma=\frac{r_{a} *(\max (X)-\min (X))}{\sqrt{8}}
$$

Una vez que han sido generadas todas las FMs tipo gaussiana, únicas posibles para ser usadas con el modelo ANFIS, se continua con la siguiente fase para la modelación difusa; en donde, la identificación de los parámetros realizada con ANFIS genera un SIL tipo Sugeno.

\subsubsection{Identificación de parámetros}

Cuando se utiliza el método de agrupamiento sustractivo y ANFIS, el SIL es de tipo Sugeno de primer orden, utiliza solamente FMs tipo gaussianas, igual número de FMs y reglas difusas (reglas tipo If - then), con una salida calculada con el método del peso promedio (valor discreto no difuso).

\subsubsection{Adaptive Neuro Fuzzy Inference System (ANFIS)}

ANFIS [7] es un modelo que combina las ventajas de redes neuronales y lógica difusa permitiendo que un SIL aprenda a través de un algoritmo híbrido que combina el método de la gradiente con el de los mínimos cuadrados. ANFIS utiliza datos existentes del tipo I/O de un sistema para tunear o pulir las FMs y crear reglas difusas para un SIL tipo Sugeno. La Fig. 5 muestra el mecanismo de inferencia lógico tipo Sugeno y la manera en que ANFIS ejecuta sus operaciones, con sus 5 capas, a fin de producir un resultado único, no difuso.

Cada capa tiene una función única a fin de procesar la información recibida hasta producir el resultado final. La capa 1 es conocida como una capa de entrada de datos y es donde se asigna un grado de membresía a cada dato utilizando las respectivas FMs gaussianas. Este proceso se lo realiza con (8).

$$
\mu\left(x_{i}\right)=e^{-\frac{1}{2}\left(\frac{x_{i}-c_{i}}{\sigma_{i}}\right)^{2}}
$$

En la capa 2 se realiza la multiplicación (П) de todos los grados de membresía que convergen al nudo para calcular los llamados "firing strengths" representados por. La capa 3 es una capa donde se normaliza cada Esto se lo consigue dividiendo cada uno para la suma total de los, obteniendo de esta manera . Luego en la capa 4, se calculan los parámetros (consecuentes) de cada función ; esto es los valores de $\mathrm{y}$, con la utilización del método de los mínimos cuadrados. Finalmente, en la capa 5 se calcula un solo resultado final () empleando el método del peso promedio. 


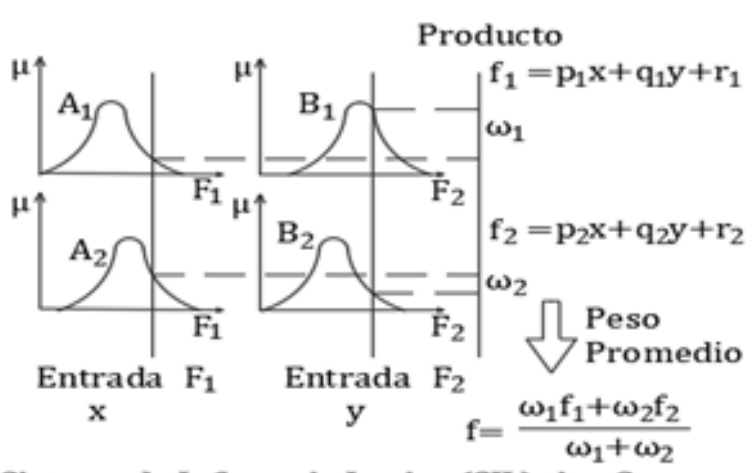

Sistema de Inferencia Logico (SIL) tipo Sugeno

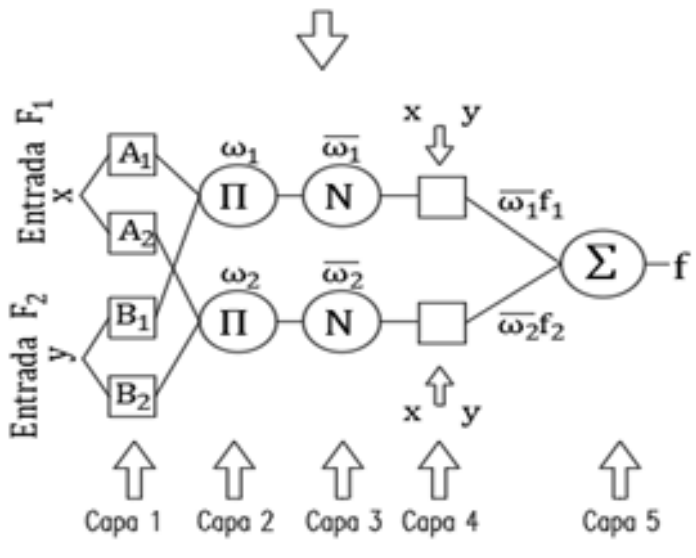

Arquitectura ANFIS

Figura 5. SIL tipo Sugeno y arquitectura ANFIS.

\section{Resultados}

El SIL para estimar el acero de refuerzo en vigas de hormigón simplemente armadas y simplemente apoyadas sometidas a cargas distribuida tiene seis variables de entrada $\left(f^{\prime} c, b, h, l u z, a, q u\right)$ y una salida $(A s)$. Los parámetros utilizados para agrupar los datos de entrada con el método de agrupamiento sustractivo fueron: relación de aceptación $(\overline{\mathcal{E}})=0.5$, relación de rechazo $(\underline{\varepsilon})=0.15$, rango de influencia $\left(r_{a}\right)=0.95 \mathrm{y}$ el factor "squash" $(\eta)=$ 1.25 , tratando de obtener el menor número posible de centros de clústeres. La Tabla 3 contiene los resultados de los cinco clústeres resultantes de la fase de identificación de la estructura que determinan los parámetros de las FMs gaussianas (ci y $\sigma i)$, mismos que fueron utilizados para obtener los grados de membresía correspondientes a cada dato de entrada.
Tabla 3. Parámetros de las funciones de membresía (FMs) gaussianas

\begin{tabular}{cccccccccccccc}
\multirow{2}{*}{$\begin{array}{c}\text { Reglas } \\
\text { Difusas }\end{array}$} & \multicolumn{3}{c}{$\mu_{f^{\prime}}$} & \multicolumn{2}{c}{$\mu_{b}$} & \multicolumn{2}{c}{$\mu_{h}$} & \multicolumn{3}{c}{$\mu_{l u z}$} & \multicolumn{2}{c}{$\mu_{a}$} & \multicolumn{2}{c}{$\mu_{q u}$} \\
\cline { 2 - 14 } & $\boldsymbol{\sigma}$ & $\mathbf{c}$ & $\boldsymbol{\sigma}$ & $\mathbf{c}$ & $\boldsymbol{\sigma}$ & $\mathbf{c}$ & $\boldsymbol{\sigma}$ & $\mathbf{c}$ & $\boldsymbol{\sigma}$ & $\mathbf{c}$ & $\boldsymbol{\sigma}$ & $\mathbf{c}$ \\
\hline 1 & 47.03 & 239.97 & 5.12 & 29.94 & 8.56 & 50.05 & 2.89 & 5.36 & 1.87 & 1.43 & 3.92 & 7.08 \\
2 & 46.98 & 300.03 & 5.08 & 34.92 & 8.40 & 59.89 & 2.39 & 7.63 & 1.54 & 1.07 & 2.14 & 7.37 \\
3 & 47.01 & 240.01 & 5.08 & 25.03 & 8.50 & 40.07 & 2.72 & 3.89 & 1.71 & 0.98 & 2.57 & 5.48 \\
4 & 47.03 & 350.00 & 5.04 & 30.13 & 8.42 & 49.98 & 1.79 & 4.86 & 0.93 & 0.24 & 2.73 & 9.99 \\
5 & 47.07 & 349.97 & 5.10 & 30.07 & 8.64 & 45.11 & 2.13 & 6.76 & 1.02 & 3.56 & 3.64 & 7.45 \\
\hline
\end{tabular}

Las seis reglas difusas del tipo condicional (If then) generadas fueron las siguientes:

1. If $\left(f^{\prime} c\right.$ está en $\left.\mu_{f^{\prime} c l}\right)$ y $\left(b\right.$ está en $\left.\mu_{b l}\right)$ y $(h$ está en $\left.\mu_{h l}\right)$ y (luz está en $\left.\mu_{l u z l}\right)$ y ( $a$ está en $\left.\mu_{a l}\right)$ y (qu está en $\mu_{q u l}$ ) then (As es $f_{1}$ )

2. If ( $f^{\prime} c$ está en $\left.\mu_{f^{\prime} c 2}\right)$ y ( $b$ está en $\left.\mu_{b 2}\right)$ y ( $h$ está en $\left.\mu_{h 2}\right)$ y $\left(l u z\right.$ está en $\left.\mu_{l u z z}\right)$ y $\left(a\right.$ está en $\left.\mu_{a 2}\right)$ y ( $q u$ está en $\left.\mu_{q u 2}\right)$ then (As es $f_{2}$ )

3. If ( $f^{\prime} c$ está en $\left.\mu_{f^{\prime} c 3}\right)$ y ( $b$ está en $\left.\mu_{b 3}\right)$ y ( $h$ está en $\left.\mu_{h 3}\right)$ y $\left(l u z\right.$ está en $\left.\mu_{\text {luzz }}\right)$ y $\left(a\right.$ está en $\left.\mu_{a 3}\right)$ y (qu está en $\left.\mu_{q u 3}\right)$ then $\left(A s\right.$ es $\left.f_{3}\right)$

4. If ( $f^{\prime} c$ está en $\left.\mu_{f^{\prime} c 4}\right)$ y $\left(b\right.$ está en $\left.\mu_{b 4}\right)$ y ( $h$ está en $\left.\mu_{h 4}\right)$ y $\left(l u z\right.$ está en $\left.\mu_{l u z}\right)$ y $\left(a\right.$ está en $\left.\mu_{a 4}\right)$ y (qu está en $\mu_{\text {qu4 }}$ ) then (As es $f_{4}$ )

5. If ( $f^{\prime} c$ está en $\left.\mu_{f^{\prime} c 5}\right)$ y ( $b$ está en $\left.\mu_{b 5}\right)$ y ( $h$ está en $\left.\mu_{h 5}\right)$ y $\left(l u z\right.$ está en $\left.\mu_{l u z 5}\right)$ y $\left(a\right.$ está en $\left.\mu_{a 5}\right)$ y (qu está en $\mu_{q u 5}$ ) then (As es $f_{5}$ )

La Tabla 4 resume los parámetros de las funciones calculados en la capa 4 cuando se utilizan seis variables. Los valores resultantes de estas funciones son multiplicados con los correspondientes para luego ser agregados para dar el resultado final.

Tabla 4. Parámetros de las funciones $f_{i}$

\begin{tabular}{cccccccc}
\hline$f_{i}$ & $\mathbf{p}$ & $\mathbf{q}$ & $\mathbf{r}$ & $\mathbf{s}$ & $\mathbf{t}$ & $\mathbf{u}$ & $\mathbf{v}$ \\
\hline 1 & 0.034 & 1.241 & 0.041 & 2.247 & -0.204 & 0.714 & -49.523 \\
2 & 0.015 & 0.063 & -0.750 & 7.785 & -2.874 & 4.959 & -25.882 \\
3 & -0.015 & -0.146 & 0.439 & 3.912 & -0.532 & 1.264 & -23.399 \\
4 & -0.081 & -3.515 & -1.390 & 12.172 & -2.609 & 4.491 & 117.927 \\
5 & 0.017 & -0.569 & -0.571 & 11.263 & -8.563 & 2.480 & -6.953 \\
\hline
\end{tabular}




\subsection{Validación del modelo}

La validación del modelo consiste en evaluar su desempeño estimando nuevos datos y comparándolos con los calculados. Para la validación del modelo ANFIS se recomienda utilizar los datos utilizados para chequear el modelo $(\mathrm{CH})$ y no los datos utilizados para entrenarlo (TR) [11]. Valores estadísticos como el coeficiente de correlación $\mathrm{R}^{2}$ y el error estándar ( $\mathrm{S}$ ), desviación estándar de los residuales, permiten determinar si un modelo se desempeña de manera adecuada $[12,13]$. La Fig. 6 representa el gráfico de valores estimados versus valores calculados, mismo que fue realizado utilizando los datos de chequeo $(\mathrm{CH})$.

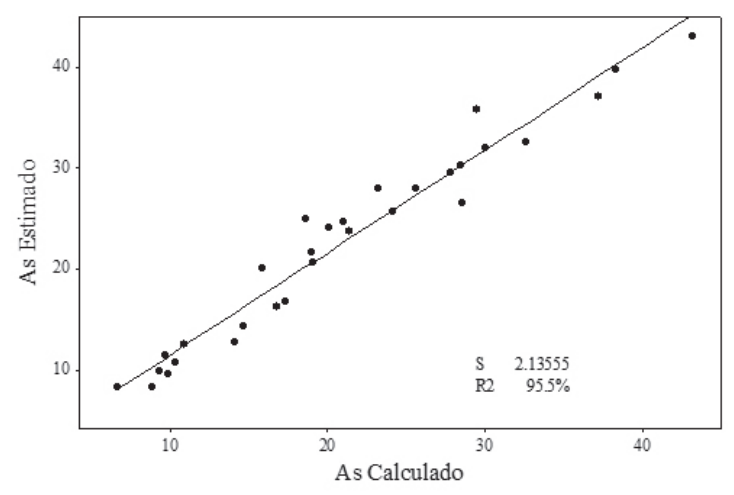

Figura 6. Valores de estimados vs. calculados.

\section{Conclusiones}

El modelo difuso tipo Sugeno resultado de este estudio, propuesto para estimar el acero de refuerzo $(A s)$ de una viga simplemente armada, de sección rectangular, simplemente apoyada, sometida a cargas distribuidas de tipo rectangular, trapezoidal y triangulares, tiene un desempeño aceptable de acuerdo a los resultados obtenidos. De la Fig. 6, se puede evidenciar que el coeficiente de correlación $\mathrm{R}^{2}$ es alto (95.5\%) los cual implica que el SIL generado es capaz de predecir o estimar nuevos datos de una manera aceptable. El valor del error $S$ calculado representa la desviación estándar de los residuales. Mientras mejor un SIL estime nuevos valores, mayor será el valor de $\mathrm{R}^{2}$ y menor será el error $S$. A pesar de que siempre va a existir un error cuando se estime el valor de $A s$, este no afectaría el diseño de una viga significativamente por cuanto el área efectiva que se coloca en el elemento estructural (área de acero real) depende de la suma de las áreas reales de acero de refuerzo disponibles en el mercado; por ejemplo, varillas de $10 \mathrm{~mm}, 12 \mathrm{~mm}$ o $14 \mathrm{~mm}$. Además, el área real de una varilla comercial no tiene el área exacta que le corresponde al diámetro respectivo debido a los resaltes propios de una varilla corrugada y a su proceso de fabricación. Generalmente se selecciona varillas comerciales que sumen un área de acero ligeramente mayor al área calculada. Lo cual sugiere que la precisión no es un parámetro determinante cuando se trata de colocar un As en una viga o cualquier elemento estructural. Se necesita, de hecho, que el valor estimado sea cercano al valor requerido (valor necesario) y, en este caso, el modelo resultante de este estudio (SIL tipo Sugeno) cumpliría satisfactoriamente con el objetivo propuesto.

El método de investigación empleado en este estudio basado en datos reales, sean estos calculados o experimentales, podría ser extendido a otras áreas del diseño de hormigón armado. Todos los códigos de construcciones, sean estos nacionales o internacionales, contemplan factores o ciertas condiciones que brindan un determinado grado de seguridad, evidenciando que los valores calculados a través de ecuaciones no son necesariamente "exactos". Es cierto que ya existen ecuaciones o funciones definidas para calcular el $A s$ en elementos estructurales; sin embargo, la teoría de lógica difusa se la podría utilizar plenamente para estimar valores, donde la exactitud no sea un determinante.

La teoría de lógica difusa brinda la posibilidad de elaborar o crear modelos, basados en lo que se conoce como conocimiento previo; esto es, información existente como son datos históricos o experimentales, a fin de ganar conocimiento de sistemas complejos a través de reglas del tipo condicional.

\section{Referencias}

[1] Ross, T. J. (2017). Fuzzy logic with engineering applications, John Wiley \& Sons, Ltd., Chichester, U.K. 
[2] Zadeh, L. A. (1965). "Fuzzy sets." Inform. Comput., 8(3), 338-353.

[3] Mamdani, E. and Assilian, S. (1975). "An experiment in linguistic synthesis with a fuzzy logic controller." Int. J. of Hum-Comp. Stud., 51(2), 135-147.

[4] Mamdani, E. (1977). "Application of fuzzy logic to approximate reasoning using linguistic synthesis." IEEE Trans. Comput., 26(12), 1182-1191.

[5] Takagi, T., and Sugeno, M. (1985). "Fuzzy identification of systems and its applications to modeling and control." IEEE Trans. Syst., Man, Cybern., 15(1), 116-132.

[6] Tsukamoto, Y. (1979). "An approach to fuzzy reasoning method", in Advances in fuzzy set theory and applications (eds Gupta, M. M. , Ragade, R. K., and Yager, R. R.), Elsevier, Amsterdam, 137-149.

[7] Jang, J.-S. R. (1993). "ANFIS: Adaptive-network-based fuzzy inference system.” IEEE Trans. Syst., Man, Cybern., 23(3), 665-685.

[8] Yager, R. R., and Filev, D. P. (1994a). Essentials of fuzzy modeling and control, Wiley, New York.
[9] Sugeno, M., and Yasukawa, T. (1993). "A fuzzy-logic-based approach to qualitative modeling." IEEE Trans. Fuzzy Syst., 1(1), 7-31.

[10] Chiu, S. L. (1994). "Prediction of fresh and hardened properties of self-consolidating concrete using neurofuzzy approach." J. Intell. Fuzzy Syst., 2, 267-278.

[11] Tesfamariam, S., and Najjaran, H. (2007). "Adaptive network-fuzzy inferencing to estimate concrete strength using mix design." J. Mater. Civil Eng., 10.1061/(ASCE)08991561(2007)19:7(550), 550-560.

[12] Tayfur, G., Erdem, T. K., and Kirca, Ö. (2014). "Strength prediction of high-strength concrete by fuzzy logic and artificial neural networks." J. Mater. Civil Eng., 10.1061/ (ASCE)MT.1943-5533.0000985, 04014079.

[13] Kostić, S., and Vasović, D. (2015). «Prediction model for compressive strength of basic concrete mixture using artificial neural networks.» Neural Comp. Appl., 26(5), 10051024. 